\title{
INFLUENCE OF VERTICAL DISPERSION AND CROSSING ANGLE ON THE PERFORMANCE OF THE LHC
}

\author{
L.H.A. Leunissen, ${ }^{*}$ CERN, Geneva, Switzerland
}

\begin{abstract}
Misalignments, magnetic field deviations and the beam crossing angle induce closed orbit deviations and residual dispersion at the interaction points of the LHC. These in turn excite synchro-betatron resonances. We present a numerical investigation of these effects and their influence on the luminosity. Bunch length effects are important and are included in the model by means of the numerical code BBC [1]. The crossing angle excites resonances that increase particle amplitudes when the tunes are near resonance while vertical dispersion changes the resonance strength. The influence on the beam core size is studied. For the nominal LHC working point, a 13th-order resonance may be crossed by the particles in the beam. The width of the synchrotron sidebands are evaluated.
\end{abstract}

\section{INTRODUCTION}

At collision energy in the LHC, local orbit bumps are used to separate the beams or make them cross at an angle. These bumps have the unwanted side effect of creating dispersion at the interaction points (IPs). Besides this, the random misalignments and imperfections of the machine generate further random components of the dispersion at the IPs. The crossing angle and dispersion in turn modify the beam-beam interaction at the IPs through the excitation of synchro-betatron resonances.

The calculations in this paper can be divided into two main classes: To study optical effects, we calculate the vertical dispersion that can be expected to arise from the sources and its effect on the beam size. For these calculations, we use the MAD [2] model of LHC Version 5 with error table 9607. Two different types of calculations are carried out:

1. Misalignments are introduced and the resulting closed orbit deviations are corrected to RMS values of $1 \mathrm{~mm}$ in both horizontal and vertical planes (simulating the typical quality of correction in a real machine).

2. The crossing angles and separation bumps are then introduced at IP1, IP2, IP5 and IP8, modifying the closed orbit again. The linear coupling is corrected in both cases again simulating the quality of correction in a real machine and the residual dispersion in the horizontal and vertical plane is calculated. In Table 1 the machine parameters of immediate interest are summarised.

To study the beam-beam effects we use the tracking program BBC [1]; it includes an appropriate model of a single

\footnotetext{
*Email: Leonardus.Leunissen@cern.ch
}

beam-beam interaction but treats the rest of the machine in linear approximation.

The dispersion at the RF cavities is assumed to be zero, so that this is not an additional source of synchro-betatron resonances.

Where it is possible to do so, the results of BBC are compared with existing analytical theory and other models. Further details are given in [3].

\begin{tabular}{|l|l|}
\hline Relativistic factor $\gamma$ & 7461 \\
Normalised emittance $\epsilon_{n}$ & $3.7510^{-6} \mathrm{~m} . \mathrm{rad}$ \\
bunch length $\sigma_{s}$ & $0.077 \mathrm{~m}$ \\
relative energy spread $\sigma_{e}$ & $1.11 \times 10^{-4}$ \\
Particles per bunch $N_{b}$ & $1.05 \times 10^{11}$ \\
Number of bunches $k_{b}$ & 2835 \\
Revolution frequency $f_{r}$ & $11245.5 \mathrm{~Hz}$ \\
$\beta^{*}(\mathrm{IP} 1, \mathrm{IP} 2, \mathrm{IP} 5, \mathrm{IP} 8)$ & $(0.5 \mathrm{~m}, 250 \mathrm{~m}, 0.5 \mathrm{~m}, 33 \mathrm{~m})$ \\
$Q_{x}, Q_{y}, Q_{s}$ & $63.31,59.32,0.00212$ \\
$Q_{x}^{\prime}, Q_{y}^{\prime}$ & $2.0,2.0$ \\
\hline
\end{tabular}

Table 1: Beam parameters of the LHC at collision energy.

\section{PARASITIC DISPERSION DUE TO THE CLOSED ORBIT}

The dispersion functions for the ideal machine are zero at the IPs. In Table 2 the horizontal and vertical dispersion are shown at the experimental interaction points together with the calculated standard deviation over the ensemble of 20 closed orbit calculations.

\begin{tabular}{|l|l|l|}
\hline I.P. & $D_{x}[\mathrm{~m}]$ & $D_{y}[\mathrm{~m}]$ \\
\hline IP1 & $-0.001 \pm 0.008$ & $-0.006 \pm 0.028$ \\
IP2 & $0.003 \pm 0.169$ & $-0.087 \pm 0.508$ \\
IP5 & $-0.002 \pm 0.009$ & $0.004 \pm 0.015$ \\
IP8 & $0.006 \pm 0.071$ & $-0.029 \pm 0.213$ \\
\hline
\end{tabular}

Table 2: Horizontal and vertical dispersion at the four experimental interaction points from residual closed orbit.

\section{PARASITIC DISPERSION DUE TO THE CROSSING ANGLE}

The dispersion created at the IPs by orbit bumps were calculated with MAD. They are presented in Table 3. 


\begin{tabular}{|l|l|l|}
\hline I.P. & $D_{x}[\mathrm{~m}]$ & $D_{y}[\mathrm{~m}]$ \\
\hline IP1 & 0.021 & -0.006 \\
IP2 & -0.087 & -0.035 \\
IP5 & -0.002 & -0.005 \\
IP8 & 0.059 & -0.028 \\
\hline
\end{tabular}

Table 3: Horizontal and vertical dispersion at the four experimental interaction points from crossing angle.

\section{LUMINOSITY CHANGE WITH THE BEAM CROSS SECTION}

Dispersion will contribute to the beam size in the following way:

$$
\left(\sigma_{z}^{*}\right)^{2}=\epsilon_{z} \beta_{z}^{*}+\left(\sigma_{e} D_{z}^{*}\right)^{2} .
$$

Here, $\sigma_{z}, D_{z}^{*}$ and $\beta_{z}^{*}$ are the beam size, the dispersion and the $\beta$-function value at the interaction point with $z \in$ $\{x, y\}$. The energy spread of the beam is denoted by $\sigma_{e}$.

The increase of the beam size leads to a change in the luminosity approximated by:

$$
L=\frac{N_{b}^{2} k_{b} f_{r}}{4 \pi \sigma_{x}^{*} \sigma_{y}^{*}} \frac{1}{\sqrt{1+\delta^{2}}} \text { and } \delta=\frac{\phi \sigma_{s}}{2 \sigma_{z}^{*}} .
$$

This equation holds when the crossing angle $\phi$ is small and the $\beta^{*}$ does not vary much near the interaction point.

Inserting Eq. 1 in Eq. 2 and then Taylor expanding with respect to $D_{y}$ shows that $\mathcal{L}$ decreases.

$$
\mathcal{L}=\mathcal{L}_{0}\left(1-\frac{2 \sigma_{e}^{2} D_{y}^{2}}{4 \epsilon_{y} \beta_{y}^{*}+\phi^{2} \sigma_{s}^{2}}+\mathcal{O}\left(D_{y}^{4}\right)\right)
$$

The design luminosity is $\mathcal{L}_{0}=10^{34} \mathrm{~cm}^{-2} \mathrm{~s}^{-1}$. The luminosity changes at most by about $5 \%$ due to the changes of horizontal and vertical beam size at the IP.

\section{RESONANCE EFFECT OF CROSSING ANGLE}

The effect of one single beam-beam interaction is studied with the program BBC. This FORTRAN code analyses a beam-beam collision with a crossing angle in a symplectic way in 6 dimensions. The bunch length effect influences the synchro-betatron resonances. To represent this, the strong beam is split into several longitudinal slices. The parameters $R=\sigma_{s} / \beta_{z}^{*}$ and $\Phi=\phi \sigma_{s} / \sigma_{z}$ (Piwinski angle) show the necessity of the slicing. For $R \geq 1$, the hourglass effect (depth of focus) [4] is important. This means that bunch length effects need to be included in the calculations by using slices. When $\Phi \geq 1$, the tilt effect is important. For the LHC $R=0.15$ and $\Phi=0.5$ (at IP1 the crossing angle is $\pm 150 \mu \mathrm{rad}$ ), the same order of magnitude. One might expect that the bunch length effects are not important, but the numerical results show that this is not the case. Calculations show that it is necessary to split the beam into five slices [3].
A separate numerical simulation was carried out to calculate the coupling of the transverse and longitudinal oscillations. This program is based on the equations of motion as derived from a Hamiltonian [5].

The tracking was done with both programs over 10000 turns. The largest amplitude during this number of turns was recorded. The resonance $q Q_{z} \pm r Q_{s}=4$ is scanned $(q=13)$ in this study.

The following plots (Figure 1 and 2) compare the maximum amplitude on the resonance as a function of the initial betatron amplitude as found by BBC calculation and the simulation with the equations of motion. Because of the symmetry of the beam-beam potential the satellites with even $q+r$ are stronger than those with odd $q+r$.

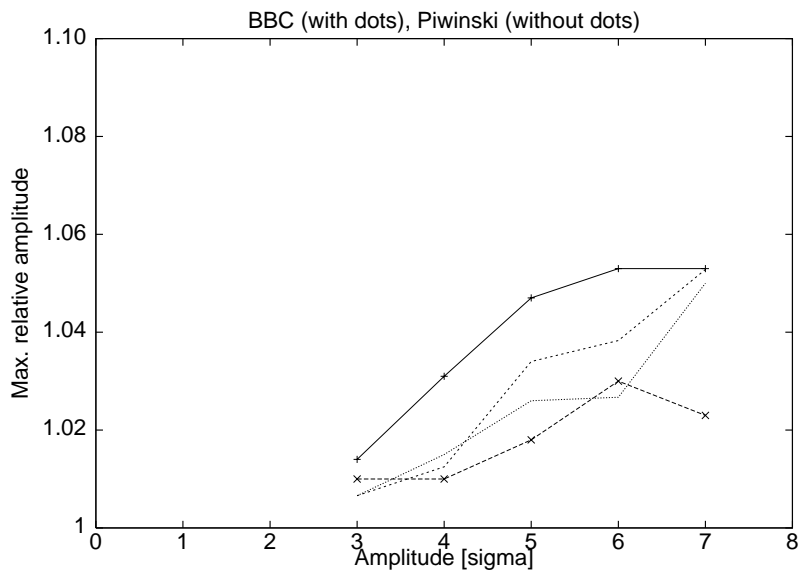

Figure 1: Resonance strength of $13 Q_{x} \pm 3 Q_{s}=4$ as a function of the initial amplitude of the particles.

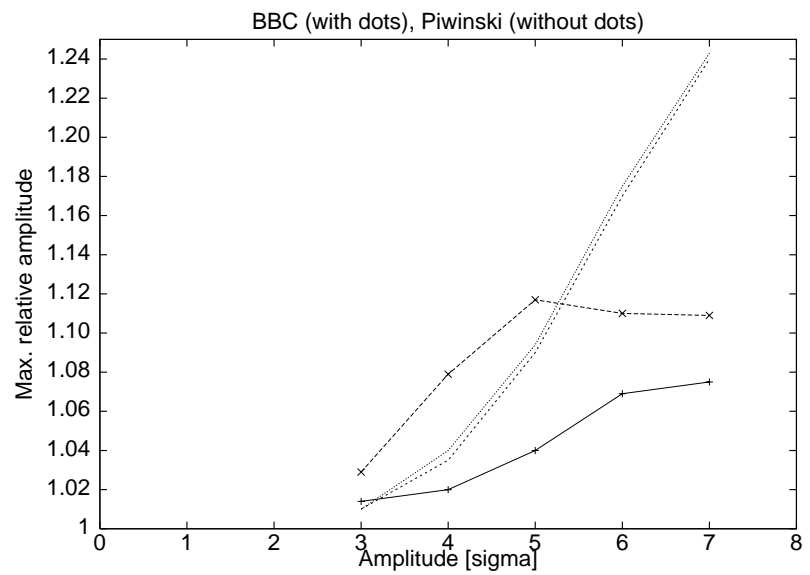

Figure 2: Resonance strength of $13 Q_{x} \pm Q_{s}=4$ as a function of the initial amplitude of the particles.

\section{RESONANCE EFFECT OF DISPERSION}

The effect of dispersion is similar to that of a crossing angle: it excites the same synchrotron satellites close to each 
beam-beam resonance. The strength of the satellites are the same in both cases when the dispersion at the IP is equal to [5]:

$$
D_{I P}=\phi \frac{\alpha_{M} \bar{R}}{2 Q_{s}}
$$

where $\alpha_{M}$ is the momentum compaction factor and $\bar{R}$ is the mean radius of the accelerator. For a dispersion of about $10 \mathrm{~cm}$ the resonances would be excited with the same strength as with the nominal crossing angle. In Figure 3 the four resonance lines described earlier $(r \in\{1,3\})$ are recalculated including a $10 \mathrm{~cm}$ vertical dispersion at the IP.

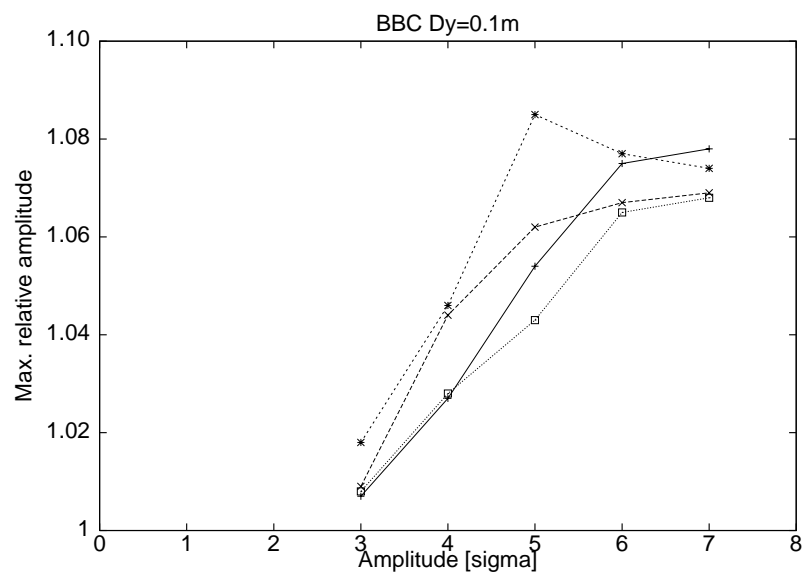

Figure 3: Resonance strength of $13 Q_{x} \pm r Q_{s}=4$ as a function of the initial amplitude of the particles with $r \in$ $\{1,3\}$ and $D_{y}=0.1 \mathrm{~m}$.

The two outer resonances become stronger and the two inner are weakened, resulting in a global equal strength of the four resonance lines. The initial amplitude is increased by about $7.5 \%$ with respect to the resonance free part.

Numerical calculations show that the width of the resonances $13 Q_{\beta} \pm 3 Q_{s}=4$ is $1 \times 10^{-4}$ (BBC). The other program yields $9 \times 10^{-5}$. The resonances $13 Q_{\beta} \pm Q_{s}=4$ have a width of about $1.5 \times 10^{-4}(\mathrm{BBC})$ whereas the other program yields $2 \times 10^{-4}$. First-order theoretical investigations [5] result in a width of the resonance islands of $6 \times 10^{-5}$.

\section{CORRECTION OF VERTICAL DISPERSION}

The closed orbit in quadrupoles and sextupoles is the driving term for the parasitic vertical dispersion. Special vertical orbit bumps can create vertical dispersion to compensate the dispersion generated by the beam crossing scheme at the IP or the closed orbit deviations. Usually a closed orbit bump starts at the beginning of one arc and is closed at the end of the same arc. To cancel the dispersion wave a second orbit bump (in the following arc) is used. In the case of a vertical dispersion of $6 \mathrm{~mm}$ (at IP1 and IP5) orbit bumps of about $2 \mathrm{~mm}$ have to be used. The correction magnets can generate kicks of at most $70 \mu \mathrm{rad}$. This generates a maximum dispersion of $2 \mathrm{~cm}$ at IP1 and IP5 (leaving the dispersion at all other IPs zero).

\section{CONCLUSIONS}

The random closed orbit deviations due to misalignments and magnetic field errors result in a few $\mathrm{cm}$ of residual dispersion at the interaction points of the LHC. The local orbit bumps in the interaction region are a further deterministic source of dispersion. This dispersion increases the beam size by less than $10 \%$.

Higher-order resonances will still be crossed by particles in the tail of the beam (large amplitudes). Their influence has been studied in detail, by tracking particles with initial amplitudes of $3,4,5$ and $6 \sigma$, both with BBC and with another program based on the equations of motion derived in [5]. Without dispersion, both methods gave similar results for the studied resonances. For example, the sidebands of the 13th order betatron resonance $\left(13 Q_{\beta} \pm r Q_{s}=4\right.$ with $\left.r \in\{1,3\}\right)$ have a width of approximately $1.5 \times 10^{-4}$. The crossing angle increases the amplitude of the particle oscillation by less than $10 \%$. BBC shows that the nominal crossing angle combined with vertical dispersion of $10 \mathrm{~cm}$ at the IP changes the strength of the resonance, giving an increase of amplitudes of $7.5 \%$ with respect to the unperturbed case. Thus, the strengths of some sidebands are reduced.

Correction of residual dispersion at the IP is possible with closed orbit bumps in the arcs. The dispersions at IP1 and IP5 can be changed independently of each other by $\pm 2 \mathrm{~cm}$ without affecting the dispersion at the other IPs. They can therefore be used to correct the dispersion arising from the beam crossing angle.

\section{REFERENCES}

[1] K. Hirata, "BBC User's Guide; A Computer Code for BeamBeam Interaction with a Crossing Angle, version 3.4", SLNote 97-57 AP (1997)

[2] H. Grote and F.C. Iselin, The MAD program (Methodical Accelerator Design) version 8.16, User's reference manual, CERN/SL/90-13(AP) (rev.4) (1995)

[3] L.H.A. Leunissen, Influence of Vertical Dispersion and Crossing Angle on the Performance of the LHC, LHC Project report, to be published

[4] G.E. Fisher, Measurements on beam-beam interaction at Spear, IEEE Trans Nucl. Sci. NS-20 (1973) p.838

[5] A. Piwinski, Limitation of the Luminosity by Satellite Resonances, DESY 77/18 (1977) 